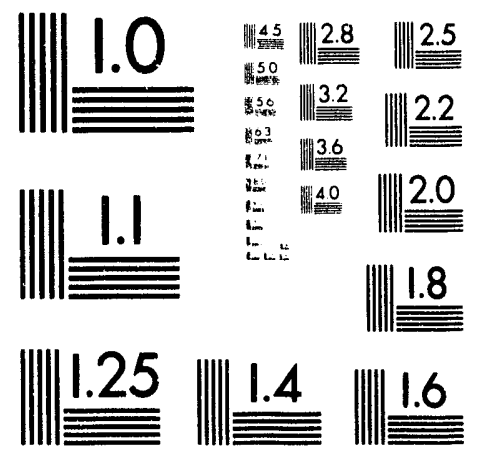



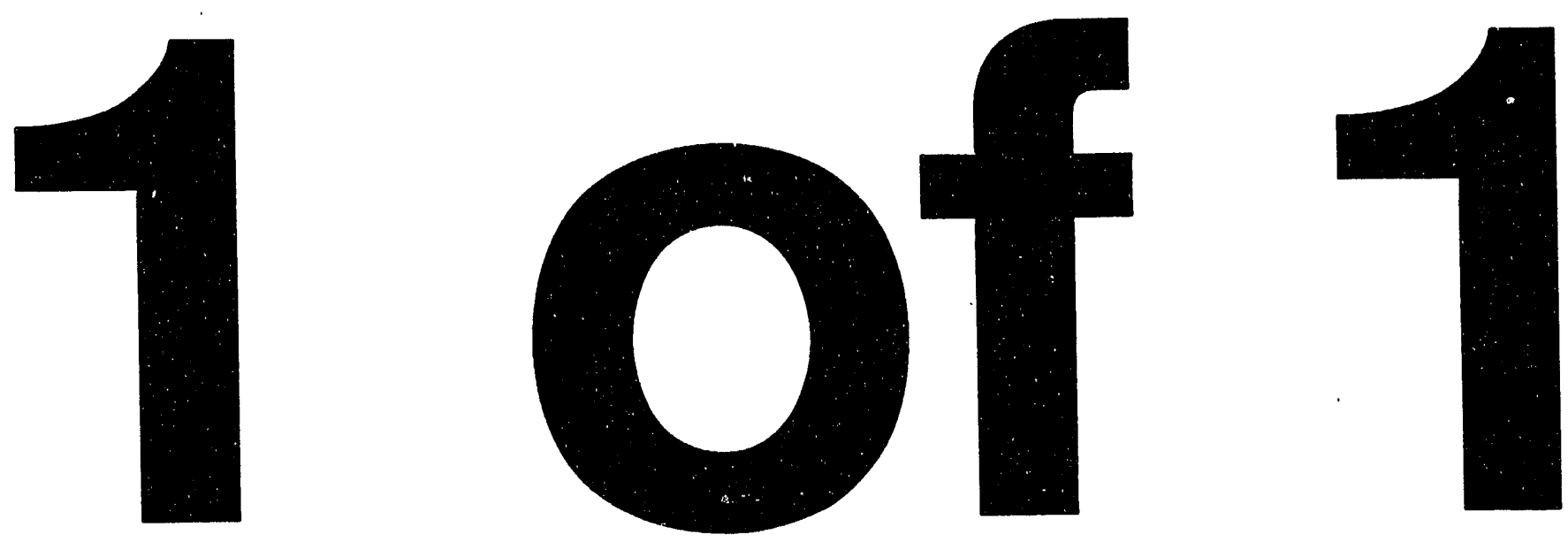
Engineering Physics and Mathematics Division

Mathematical Sciences Section

\title{
A COMPARISON OF ITERATIVE METHODS FOR A MODEL COUPLED SYSTEM OF ELLIPTIC EQUATIONS
}

June M. Donato

\begin{abstract}
Mathematical Sciences Scction
Oak Ridge National Laboratory

P.O. Box 2008, Bldg. 6012

Oak Ridge, TN 37831-6367
\end{abstract}

Date Published: August 1993

Research was supported by the Applied Mathematical Sciences Research Program of the Office of Energy Research, U.S. Department of Energy.

Prepared by the

Oak Ridge National Laboratory

Oak Ridge, Tennessee 37831

managed by

Martin Marietta Energy Systems, Inc.

for the

U.S. DEPARTMENT OF ENERGY. under Contract No. DE-AC05-84OR21400 


\title{
A COMPARISON OF ITERATIVE METHODS FOR A MODEL COUPLED SYSTEM OF ELLIPTIC EQUATIONS
}

\author{
June M. Donato
}

\begin{abstract}
Many interesting areas of current industry work deal with non-linear coupled systems of partial differential equations. We examine iterative methods for the solution of a model two-dimensional coupled system based on a linearized form of the two carrier drift-diffusion equations from semiconductor modeling. Discretizing this model system yields a large non-symmetric indefinite sparse matrix.

To solve the model system various point and block methods, including the hybrid iterative method Alternate Block Factorization (ABF), are applied. We also employ GMRES with various preconditioners, including block and point incomplete LU (ILU) factorizations.

The performance of these methods is compared. It is seen that the preferred ordering of the grid variables and the choice of iterative method are dependent upon the magnitudes of the coupling parameters. For this model, ABF is the most robust of the non-accelerated iterative methods. Among the preconditioners employed with GMRES, the blocked "by grid point" version of both the ILU and MILU preconditi ners are the most robust and the most time efficient over the wide range of parameter values tested. This information may aid in the choice of iterative methods and preconditioners for solving more complicated, yet analogous, coupled systems.
\end{abstract}




\section{Introduction}

Scalar elliptic and systems of coupled elliptic partial differential equations (PDEs) arise frequently in the modeling of physical processes. Examples include the steady-state equation for heat conduction and the steady-state drift-diffusion equations that appear in semiconductor modeling. We will consider a variety of iterative methods including Alternate-Block-Factorization (ABF) and the GMRES method with a number of preconditioners. We will be particularly interested in comparing the performance of point and block methods in solving a model coupled system.

Let us consider such a coupled system of equations on a region $\Omega \subset \mathbf{R}^{\mathbf{n}}$ which can be written in the form

$$
L_{1}\left(v^{(1)}, \ldots, v^{(m)}\right)=0, \ldots, L_{m}\left(v^{(1)}, \ldots, v^{(m)}\right)=0
$$

with specified conditions on the boundary of $\Omega$. The $v^{(j)}$ are the variables of interest and the $L_{i}$ are known, possibly nonlinear, functions of the $v^{(j)}$. Each constituent $v^{(j)}$ can be viewed as the concentration of a given species in reaction with the other species according to the above system. In semiconductor modeling, for example, $v^{(1)}, v^{(2)}$, and $v^{(3)}$ would represent the electrostatic potential, the electron density, and the hole density, respectively.

If the $L_{i}$ are nonlinear in the $v^{(j)}$, a typical method of solution is nonlinear GaussSeidel (also known as Gummel's iteration).

In other methods, such as the Gauss-Seidel Newton method, the equations are first linearized. The resulting system of linear equations is then discretized on a partitioning of $\Omega$ to obtain a matrix system

$$
\mathcal{A} \underline{v}=\underline{b}
$$

where $\underline{v}=\left(\underline{v}^{(1)}, \ldots, \underline{v}^{(m)}\right)^{t}$ and $\underline{v}^{(j)}=\left(v_{1}^{(j)}, v_{2}^{(j)}, \ldots, v_{N}^{(j)}\right)^{t}$ is a column vector representing the values of the function $v^{(j)}$ at the $N$ grid points of $\Omega$. Hence, at a given grid point, there are $m$ values to be calculated, one for each species $v^{(j)}$. The matrix $\mathcal{A}$ is typically very large and sparse.

Our goal is to find efficient methods for solving such a matrix system. Certainly, we could use direct solvers. But for large sparse systems, especially for three-dimensional problems, these methods may become prohibitive in both arithmetic complexity and memory requirements and hence in cost and time.

Iterative methods, however, are well suited for the solution of large sparse linear systems. Jacobi, Gauss-Seidel, Successive-Overrelaxation (SOR) and other iterative methods and accelerators are well established $[6,7,10,12,13]$. They can easily be implemented to take full advantage of sparsity patterns of the matrix in order to conserve on both storage and computing time.

For large, sparse, coupled systems of equations, the choice of an iterative method also depends on the coupling between the unknown variables $v^{(j)}$ [2]. This coupling suggests the use of different reorderings of the dependent variables which in turn may lead to different preconditioners than result from the original ordering.

For example, in the system (1) $\mathcal{A} \underline{v}=\underline{b}$, the ordering could be done "by equation" where the grid $N$ values for the constituent $v^{(1)}$ occur first, followed by those for $v^{(2)}$, 
and so on for each of the $m$ variables $v^{(j)}$. So the vector $\underline{v}$ has the form

$$
\underline{v}=\left(v_{1}^{(1)}, v_{2}^{(1)}, \ldots, v_{N}^{(1)}, v_{1}^{(2)}, \ldots, v_{N}^{(2)}, \ldots, v_{1}^{(m)}, \ldots, v_{N}^{(m)}\right)^{t} .
$$

An alternative ordering is "by grid point" where first we order all the values of the constituents at grid point 1 , then those values at grid point 2 , and so forth for each of the $N$ grid points. This permuted vector $\underline{\tilde{v}}$ of $\underline{v}$ would look like

$$
\underline{\tilde{v}}=\left(v_{1}^{(1)}, v_{1}^{(2)}, \ldots, v_{1}^{(m)}, v_{2}^{(1)}, \ldots, v_{2}^{(m)}, \ldots, v_{N}^{(1)}, \ldots, v_{N}^{(m)}\right)^{t} .
$$

Here we investigate certain point and block iterative methods and preconditioners, especially those based on the orderings "by equation" and "by grid point."

The rest of this paper is organized as follows. In section 2 the model coupled system is presented in both "by equation" and "by grid point" ordering. In section 3 the methods utilized for solving the model are overviewed. In section 4 experimental results are presented and discussed. In section 5 the main conclusions are summarized.

\section{The Model Coupled System}

In this study of coupled systems of equations, we are motivated by the steady-state normalized two carrier drift-diffusion equations from semiconductor modeling

$$
\begin{array}{r}
-\Delta u+v-p-N(x)=0 \\
\nabla \cdot(v \nabla u-\nabla v)=0 \\
\nabla \cdot(p \nabla u-\nabla p)=0 .
\end{array}
$$

The functions $u, v, p$, and $N(x)$ represent the electrostatic potential, the density of electrons, the density of holes, and the doping profile, respectively.

To attack this set of nonlinear equations with numerical linear algebra techniques, the equations are reduced in the following manner:

1. Consider only the one carrier system. (Only $u$ and $v$ are kept in the equations.)

2. Linearize the resulting equations.

3. Assume that "the response of carriers to a change in the electric field is much faster than the effective rate of change in the field." In other words, assume that $\nabla v>\nabla u$. (This assumption is not unusual, see [3].)

This yields the following pair of linear second order coupled elliptic equations in $u$ and $v$ :

$$
\begin{aligned}
-\Delta u+v & =f, \\
-\Delta v+\vec{\epsilon} \cdot \nabla v+\eta \Delta u & =g \text { on } \Omega .
\end{aligned}
$$

The positive real parameters $\epsilon$ and $\eta$ result from the linearization step. The constant $\epsilon$ is an approximation to the magnitude of $\nabla u$ in comparison to $\nabla v, \eta$ is an approximation for $v$ in comparison to $\nabla u$, and $\vec{\epsilon}=(\epsilon, \epsilon)$. For this model problem, we use Dirichlet 
boundary conditions and let $\Omega=[0,1] \times[0,1]$. The one-dimensional version of this system has been examined in [2].

The usual five-point stencil discretization of the Laplacian with the natural rowwise ordering on an $n^{2} \times n^{2}$ mesh is used with uniform grid spacing $h=\frac{1}{n+1}$. The resulting scaled $2 \times 2$ block system with $n \times n$ subblock is

$$
\mathcal{A} w=b
$$

where

$$
\begin{aligned}
& \mathcal{A}=h^{2}\left(\begin{array}{cc}
-\Delta_{h} & I \\
\eta \triangle_{h} & -\Delta_{h}+\epsilon \nabla_{h}
\end{array}\right)=\left(\begin{array}{cc}
\triangle_{5} & h^{2} I \\
-\eta \triangle_{5} & \Delta_{5}+\epsilon^{\prime} S
\end{array}\right) \in R^{2 n^{2} \times 2 n^{2}}, \\
& \epsilon^{\prime}=\epsilon h \\
& \triangle_{5}=-h^{2} \triangle_{h}=\left[\begin{array}{rrr}
-1 & \cdot \\
-1 & 4 & -1 \\
\cdot & -1 & \cdot
\end{array}\right] \in R^{n^{2} \times n^{2}}, \\
& \boldsymbol{w}=\left(\begin{array}{l}
u \\
v
\end{array}\right) \\
& \boldsymbol{b}=h^{2}\left(\begin{array}{c}
f \\
g
\end{array}\right)=\left(\begin{array}{c}
\tilde{f} \\
\tilde{g}
\end{array}\right) .
\end{aligned}
$$

Upwind differencing is used to approximate the $\vec{\epsilon} \cdot \nabla v$ term of the equation (2), so that

$$
\epsilon^{\prime} S=\epsilon h^{2} \nabla_{h}=(\epsilon h)\left(h \nabla_{h}\right)=\epsilon^{\prime}\left[\begin{array}{ccc} 
& \cdot & \\
-1 & 2 & . \\
& -1 &
\end{array}\right]
$$

In the above system, "by equation" ordering was used where all the grid point values for $u$ are ordered prior to those for $v$. For example,

$$
w=\left(\begin{array}{l}
u \\
v
\end{array}\right)=\left(u_{11}, u_{12}, \ldots, u_{n n}, v_{11}, \ldots, v_{n n}\right)^{t} .
$$

The discretized system can be ordered in a variety of ways. In particular, we are interested in studying the ordering "by grid point" where the values of $u$ and $v$ on a given grid point are taken as a pair and the natural rowwise ordering is used for these pairs. For this problem this yields

$$
\tilde{w}=\left(u_{11}, v_{11}, \ldots, u_{n n}, v_{n n}\right)^{t}
$$

The system would then appear as

$$
\tilde{\mathcal{A}} \tilde{w}=\tilde{b}
$$


where $\tilde{\mathcal{A}}$ is an $n \times n$ block pentadiagonal matrix with $2 \times 2$ subblocks,

$$
\tilde{\mathcal{A}}=\left[\begin{array}{rrr}
\cdot & B & \cdot \\
C & A & B \\
\cdot & C & \cdot
\end{array}\right],
$$

where

$$
A=\left(\begin{array}{cc}
4 & h^{2} \\
-4 \eta & 4+2 \epsilon^{\prime}
\end{array}\right), \quad B=\left(\begin{array}{cc}
-1 & 0 \\
\eta & -1
\end{array}\right) \text {, and } \quad C=\left(\begin{array}{cc}
-1 & 0 \\
\eta & -1-\epsilon^{\prime}
\end{array}\right) .
$$

Notes that there is a permutation matrix $\mathcal{P}$ such that

$$
\tilde{\mathbb{A}}=\mathcal{P} \mathcal{A} \mathcal{P}^{t}
$$

\section{The Methods}

The model system is difficult to analyze directly due to the convection term. However, in [5], two simpler model problems were examined that lent themsel'res to analytic techniques. Analysis for one of these models, an indefinite non-singular coupled system, was used in predicting which methods would be effective for the model presented here. Information gleaned from the analysis was utilized in the choice of methods to be included for study with this current moriel system. As we shall show later, the incomplete factorization preconditioners based on ordering "by grid point" were the most robust and typically the most time efficient methods. The robustness of these preconditioners correlated well with the analysis given in reference [5] for the indefinite non-singular coupled system.

Note that it is feasible to solve the model system (2) by solving for $v$ in the first equation and then substituting into the second equation yielding a single fourth order equation in $u$. However, the goal is to determine iterative methods and preconditioners which will be "good" for more complicated yet analogous coupled systems that do not lend themselves to such an approach.

Here we investigate certain point and block methods, especially those based on the orderings "by equation" and "by grid point." Once an ordering has been selected, methods can be implemented in a point or block fashion. In a point method, one element at a time is updated. In a block method, the variables are ordered into groups and variables within the same group are updated as a unit.

Methods for (3) lend themselves to $2 \times 2$ block methods where the subblocks are $n^{2} \times n^{2}$ matrices. These block methods will be called "block equation" (BE) methods.

Methods considered for (5) will be $n^{2} \times n^{2}$ block methods where the subblocks are $2 \times 2$ matrices. These methods will be designated "block grid" (BG) methods.

In either ordering, we can chose to use point iterative methods. However, using point methods in the "by equation" ordering (PE - point equation) and point methods in the "Jy grid point" ordering (PG - point grid) did not yield significant, if any, difference in results. So, we only present results for the PE, BE and BG implementations of the methods. 
To solve the model problem the following methods were employed: Jacobi and Gauss-Seidel iterative methods, Alternate-Block-Factorization (ABF), and the Krylov subspace method GMRES [11] with Jacobi, Gauss-Seidel, and incomplete LU preconditioners.

Background on $A B F$ is presented in [2]. It is a hybrid (composite) method in that both "by grid point" and "by equation" orderings are used: a block Gauss-Sejdel iteration is applied to the matrix $\mathcal{A}$ in "by equation" ordering after it has been postconditioned by the block Jacobi splitting matrix for $\tilde{\mathcal{A}}$ in "by grid point" ordering.

The remaining methods and preconditioners are fairly standard and further background can be found in $[6,7,11,12,13,9]$.

The incomplete $\mathrm{LU}$ preconditioners are presented using the block $(2 \times 2)$ versions in "grid point" ordering. The point-equation version did not exist or were unstable for this model, so such data is not presenced. The block version using "by equation" ordering does not make sense with this model, since no $n^{2} \times n^{2}$ block is fully zero, there would be no advantage in terms of sparsity.

For some block methods, when ordering is done "by equation," an inner iterative technique may be necessary for the sub-solves. GMRES is employed as the 'inner' solver in these cases. When ordering is done "by grid point," the 'inner' solves are done exactly.

Notation The shorthand denotation of the methods studied herein is as follows. The main letter denotes the method or preconditioner, the first subscript denotes whether the method is implemented in a point or block form, and the second subscript indicates whether the ordering was "by equation" or "by grid point." For example, $\mathrm{J}_{P E}$ stands for the typical point-Jacobi method on $\mathcal{A}$ (in "by equation" ordering). $\mathrm{GS}_{B G}$ stands for the Gauss-Seidel method implemented in block $(2 \times 2)$ form in the "by grid point" ordering.

\section{Experimental Results}

The experimental results are based on the solution of

$$
\mathcal{A}\left(\begin{array}{l}
u \\
v
\end{array}\right)=\left(\begin{array}{c}
f \\
g
\end{array}\right),
$$

where the right-hand-sides $f$ and $g$ are chosen so that the true solutions for $u$ and $v$ are given by

$$
\begin{aligned}
& u(x, y)=32 x^{2}(x-1) y\left(y^{2}-1\right) \\
& v(x, y)=16 x(1-x) y(1-y)
\end{aligned}
$$

The model equations were discretized on an $n \times n$ grid. Hence, $u$ and $v$ represent $N=n^{2}$ length column vectors and $\mathcal{A}$ is an order $2 N$ matrix. For $n=7$ there are 98 unknowns, and for $n=15$ there are 450 unknowns.

The codes were written in Fortran 77 using double precision floating-point arithmetic. The experiments were all run on a dedicated Sun 3-280. The stopping criterion 
Table 1: Iterations (Time) for $n=7, \eta=0$

\begin{tabular}{|c|c|c|c|c|c|c|c|c|c|c|}
\hline Iter & \multicolumn{2}{|c|}{$\epsilon=0$} & \multicolumn{2}{|c|}{$\epsilon=1$} & \multicolumn{2}{|c|}{$\epsilon=10$} & \multicolumn{2}{|c|}{$\epsilon=50$} & \multicolumn{2}{|c|}{$\epsilon=100$} \\
\hline$J_{P E}$ & & $\begin{array}{ll}- \\
-\end{array}$ & & - & & - & & - & & - \\
\hline$\overline{G S} S_{P E}$ & & - & & 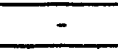 & & - & & - & & - \\
\hline$J_{B E}$ & 3 & $(2.48)$ & 3 & $(3.66)$ & 3 & $(3.20)$ & 3 & $(2.98)$ & 3 & $(2.98)$ \\
\hline$G S_{B E}$ & & $(3.06)$ & 3 & $(3.56)$ & 3 & $(3.18)$ & 3 & $(2.98)$ & 3 & $(2.90)$ \\
\hline$J_{B G}$ & & - & & - & & $=$ & & - & & - \\
\hline$\overline{G S} S_{B G}$ & & - & & - & & - & & - & & - \\
\hline$A B F$ & & $(3.02)$ & & $(3.52)$ & & $(3.16)$ & & $(2.90)$ & & $(2.82)$ \\
\hline Precond & & $=0$ & & $=1$ & & $=10$ & & $=50$ & & 100 \\
\hline$J_{P E}$ & 35 & $(4.56)$ & 44 & $(5.70)$ & & $(8.34)$ & 72 & $(9.34)$ & 71 & $(9.16)$ \\
\hline$\overline{G S} S_{P E}$ & 28 & $(4.74)$ & 30 & $(5.08)$ & 26 & $(4.44)$ & 22 & (3.72) & 21 & (3.64) \\
\hline$J_{B E}$ & 2 & $(4.32)$ & & 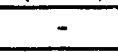 & 2 & $(4.94)$ & 2 & $(4.68)$ & 2 & $(4.64)$ \\
\hline$G S_{B E}$ & 2 & $(3.86)$ & & - & $\overline{2}$ & $(5.02)$ & 2 & $(4.64)$ & 2 & $(4.66)$ \\
\hline$J_{B G}$ & $\overline{34}$ & $(4.50)$ & 47 & $(6.20)$ & 70 & $(9.20)$ & 71 & $(9.32)$ & 72 & $(9.46)$ \\
\hline $\bar{G} S_{B G}$ & 27 & $(3.90)$ & 28 & $(4.04)$ & 26 & $(3.76)$ & 22 & $(3.20)$ & 21 & $(3.02)$ \\
\hline & 13 & $(2.72)$ & 12 & $(2.54)$ & 10 & $(2.12)$ & 10 & (2.12) & 10 & $(2.12)$ \\
\hline$M I L U_{B C}$ & 11 & $(2.30)$ & 11 & $(2.30)$ & 11 & $(2.32)$ & 10 & $(2.10)$ & 10 & $(2.18)$ \\
\hline
\end{tabular}

for convergence in all cases was for the ratio of the current residual to the initial residual to be less than $10^{-7}$.

Iteration counts and computational times (in parentheses) for the iterative methods and preconditioned GMRES are given for the model for a set of values for $\epsilon$ given a value of $\eta$. Tables $1,2,3,4$, and 5 , correspond to values of $\eta=0,1,10,50$, and 100, respectively.

By examining the performance of the methods in these tables, we see that few methods converge for all the combination of parameter values.

The only methods that converge throughout are ABF and GMRES preconditioned by $J_{B G}, G S_{B G}, I L U_{B G}$, and $M I L U_{B G}$. Hence, these methods are seen to be are the most robust. In addition, among these methods the $I L U_{B G}$ and $M I L U_{B G}$ preconditioners with GMRES are the most time efficient. 
Table 2: Iterations (Time) for $n=7, \eta=1$

\begin{tabular}{|c|c|c|c|c|c|c|c|c|c|c|}
\hline Iter & \multicolumn{2}{|r|}{$\epsilon=0$} & \multicolumn{2}{|c|}{$\epsilon=1$} & \multicolumn{2}{|c|}{$\epsilon=10$} & \multicolumn{2}{|c|}{$\epsilon=50$} & \multicolumn{2}{|c|}{$\epsilon=100$} \\
\hline$J_{P E}$ & & - & & - & & - & & - & & - \\
\hline$G S_{P E}$ & & - & & - & & - & & - & & - \\
\hline$J_{B E}$ & & - & & - & 35 & $(26.90)$ & 19 & $(14.66)$ & 15 & $(11.58)$ \\
\hline$G S_{B E}$ & & - & & $\overline{-}$ & 11 & $(9.40)$ & & $(5.96)$ & & $(6.00)$ \\
\hline$J_{B G}$ & & - & & - & & - & & - & & - \\
\hline$\overline{G S} S_{B G}$ & & - & & - & & - & & - & & - \\
\hline$A B F$ & 3 & $(3.04)$ & & $(13.68)$ & 13 & $(10.50)$ & 9 & $(7.14)$ & & $(5.66)$ \\
\hline Precond & & $\epsilon=0$ & & $\epsilon=1$ & & $=10$ & & $=50$ & & $=100$ \\
\hline$J_{P E}$ & & - & & - & & - & 91 & $(11.74)$ & 69 & $(8.90)$ \\
\hline$G S_{P E}$ & & $(13.60)$ & & - & 39 & $(6.62)$ & 26 & $(4.46)$ & 22 & $(3.78)$ \\
\hline$J_{B E}$ & & - & & - & 23 & $(39.44)$ & 11 & $(18.42)$ & 8 & $(13.82)$ \\
\hline$G S_{B E}$ & & - & & - & 5 & $(9.96)$ & 4 & $(7.80)$ & 4 & $(7.74)$ \\
\hline$J_{B G}$ & 29 & $(3.88)$ & 39 & $(5.20)$ & 81 & $(10.70)$ & 74 & $(9.76)$ & 77 & $(10.20)$ \\
\hline$G S_{B G}$ & 27 & $(3.92)$ & 29 & $(4.16)$ & 28 & $(4.08)$ & 28 & $(4.10)$ & 27 & $(3.94)$ \\
\hline$I L U_{B G}$ & 10 & $(2.14)$ & 11 & $(2.34)$ & 11 & $(2.32)$ & 10 & $(2.14)$ & 10 & $(2.14)$ \\
\hline$M I L U_{B G}$ & 11 & $(2.34)$ & 11 & $(2.32)$ & 11 & $(2.32)$ & 11 & $(2.32)$ & 10 & $(2.14)$ \\
\hline
\end{tabular}

Table 3: Iterations (Time) for $n=7, \eta=10$

\begin{tabular}{|c|c|c|c|c|c|c|c|c|c|}
\hline Iter & \multicolumn{2}{|c|}{$\epsilon=0$} & \multicolumn{2}{|r|}{$\epsilon=1$} & \multicolumn{2}{|c|}{$\epsilon=10$} & \multicolumn{2}{|c|}{$\epsilon=50$} & $\epsilon=100$ \\
\hline$\overline{J_{P E}}$ & & - & & - & & - & & - & - \\
\hline$G S_{P E}$ & & $\overline{-}$ & & - & & - & 83 & $(4.46)$ & $(4.64)$ \\
\hline$J_{B E}$ & & - & & - & & - & 51 & $(36.04)$ & $(25.74)$ \\
\hline$G S_{B E}$ & & - & & - & & - & 13 & $(10.34)$ & $(7.36)$ \\
\hline$\overline{J_{B G}}$ & & - & & - & & $=$ & & - & - \\
\hline$\overline{G S_{B G}}$ & & - & & - & & - & & - & - \\
\hline$A B F$ & 3 & $(2.34)$ & 15 & $(9.36)$ & 25 & $(16.90)$ & & $(11.08)$ & $11 \quad(8.22)$ \\
\hline$\overline{\text { Precond }}$ & & $\varepsilon=0$ & & $\epsilon=1$ & & $=10$ & & $=50$ & $\epsilon=100$ \\
\hline$J_{P E}$ & & - & & - & & - & & - & $90 \quad(11.60)$ \\
\hline$G S_{P E}$ & & $(15.42)$ & & - & $\overline{52}$ & $(8.80)$ & 34 & $(5.80)$ & $(5.28)$ \\
\hline$\overline{J_{B E}}$ & & - & & - & & - & & - & $(130.60)$ \\
\hline$\overline{G S} S_{B E}$ & & - & & - & & - & & - & - \\
\hline$J_{B G}$ & 22 & $(2.98)$ & 33 & $(4.42)$ & 49 & $(6.50)$ & 69 & $(9.12)$ & $(10.92)$ \\
\hline$G S_{B G}$ & 28 & $(4.06)$ & & $(4.08)$ & 28 & $(4.12)$ & 28 & $(4.04)$ & $(4.06)$ \\
\hline$I L U_{\boldsymbol{B} G}$ & 10 & $(2.14)$ & 10 & $(2.22)$ & 10 & $(2.12)$ & 10 & $(2.14)$ & $(2.14)$ \\
\hline$M I L U_{B G}$ & 11 & $(2.32)$ & 11 & $(2.32)$ & 11 & $(2.34)$ & 11 & $(2.32)$ & $(2.36)$ \\
\hline
\end{tabular}


Table 4: Iterations (Time) for $n=7, \eta=50$

\begin{tabular}{|c|c|c|c|c|c|c|c|c|c|c|}
\hline Iter & \multicolumn{2}{|c|}{$\epsilon=0$} & \multicolumn{2}{|r|}{$\epsilon=1$} & \multicolumn{2}{|c|}{$\epsilon=10$} & \multicolumn{2}{|c|}{$\epsilon=50$} & \multicolumn{2}{|c|}{$\epsilon=100$} \\
\hline$J_{P E}$ & & - & & - & & - & & - & & - \\
\hline$\overline{G S} S_{P E}$ & & $=$ & & $\overline{-}$ & & - & & - & 83 & $(4.40)$ \\
\hline$J_{B E}$ & & - & & - & & - & & - & & - \\
\hline$\overline{G S} S_{B E}$ & & - & & - & & - & 87 & $(55.84)$ & 25 & $(18.16)$ \\
\hline$J_{B G}$ & & - & & - & & - & & - & & - \\
\hline$\overline{G S} S_{B G}$ & & - & & - & & - & & - & & - \\
\hline$A B F$ & 3 & $\overline{(2.16)}$ & $\overline{9}$ & $(5.26)$ & & $(11.70)$ & 23 & $(15.00)$ & 19 & $(12.74)$ \\
\hline Precond & & $=0$ & & $\varepsilon=1$ & & $=10$ & & $=50$ & & $=100$ \\
\hline$J_{P E}$ & & - & & - & & - & & - & & - \\
\hline$G S_{P E}$ & & - & & $(15.46)$ & & $\overline{(10.62)}$ & 43 & $(7.34)$ & 38 & $(6.46)$ \\
\hline$J_{B E}$ & & - & & - & & $-\overline{-}$ & & - & & - \\
\hline$\overline{G S} S_{B E}$ & & - & & - & & - & & - & & - \\
\hline$J_{B G}$ & & $(2.84)$ & 24 & $\overline{(3.22)}$ & 70 & $(9.30)$ & 51 & $(6.82)$ & 57 & $(7.76)$ \\
\hline$G S_{B G}$ & & $(4.08)$ & 28 & $(4.06)$ & 28 & $(4.08)$ & 28 & $(4.04)$ & 28 & $(4.06)$ \\
\hline$I L U_{B G}$ & & $\overline{(2.14)}$ & 10 & $(2.16)$ & 10 & $(2.16)$ & 10 & $(2.16)$ & 10 & $(2.14)$ \\
\hline$\overline{M I L U_{B G}}$ & 11 & $(2.32)$ & 11 & $(2.32)$ & & $(2.36)$ & 11 & $(2.42)$ & 11 & $(2.34)$ \\
\hline
\end{tabular}

Table 5: Itcrations (Time) for $n=7, \eta=100$

\begin{tabular}{|c|c|c|c|c|c|}
\hline Iter & $\epsilon=0$ & $\epsilon=1$ & $\epsilon=10$ & $\epsilon=50$ & $\epsilon=100$ \\
\hline$\overline{J_{P E}}$ & - & - & - & - & - \\
\hline$\overline{G S} S_{P E}$ & - & - & - & - & - \\
\hline$J_{B E}$ & - & - & - & - & - \\
\hline$\overline{G S_{B E}}$ & - & - & - & - & $\begin{array}{ll}79 \quad(50.60) \\
\end{array}$ \\
\hline$J_{B G}$ & - & - & - & - & - \\
\hline$G S_{B G}$ & - & - & - & - & - \\
\hline$A B F$ & $\begin{array}{ll}3 & (2.02) \\
\end{array}$ & $7 \quad(4.32)$ & $15 \quad(8.94)$ & $\begin{array}{ll}23 & (14.30) \\
\end{array}$ & $21 \quad(13.72)$ \\
\hline Precond & $\epsilon=0$ & $\epsilon=1$ & $\epsilon=10$ & $\epsilon=50$ & $\epsilon=100$ \\
\hline$J_{P E}$ & - & - & - & - & - \\
\hline$G S_{P E}$ & $=$ & - & $94 \quad(15.82)$ & $50 \quad(8.44)$ & $41 \quad(6.96)$ \\
\hline$J_{B E}$ & - & - & - & - & - \\
\hline$\overline{G S} S_{B E}$ & - & - & - & - & - \\
\hline$J_{B G}$ & $(2.72)$ & $(3.12)$ & $(5.84)$ & $\begin{array}{ll}40 & (5.34)\end{array}$ & $(6.74)$ \\
\hline$G S_{B G}$ & $(4.12)$ & $(4.06)$ & $(4.06)$ & $(4.06)$ & $(4.06)$ \\
\hline$I L U_{B G}$ & $(2.12)$ & $(2.16)$ & $(2.14)$ & $(2.12)$ & $(2.14)$ \\
\hline$\overline{M I L U_{B G}}$ & $(2.36)$ & $(2.34)$ & $\overline{(2.32)}$ & $(2.34)$ & $(2.34)$ \\
\hline
\end{tabular}


Results for $n=15$

We have seen from the $n=7$ experimental results that the most robust methods for the model were ABF and GMRES preconditioned by $J_{B G}, G S_{B G}, I L U_{B G}$ and $M I L U_{B G}$. Hence, only the data for these five methods have been tabulated in Tables 6 and 7.

As in the previous tables, these two tables include the iteration counts and computational time for $\epsilon=0,1,10,50$, and 100 for the set of $\eta$ values of $0,1,10,50$, and 100.

From these results, we see again that $I L U_{B G}$ and $M I L U_{B G}$ are the most efficient in time.

Table 6: Iterations (Time) for $n=15, \eta=0,1,10$, varying $\epsilon$

\begin{tabular}{|c|c|c|c|c|c|c|c|c|c|c|}
\hline$\eta=0$ & \multicolumn{2}{|c|}{$\epsilon=0$} & \multicolumn{2}{|c|}{$\epsilon=1$} & \multicolumn{2}{|c|}{$c=10$} & \multicolumn{2}{|c|}{$t=50$} & \multicolumn{2}{|c|}{$\epsilon=100$} \\
\hline$A B F$ & 3 & $(38.60)$ & $\overline{3}$ & $(44.94)$ & 3 & $(49.96)$ & 3 & $(53.12)$ & 3 & $(58.34)$ \\
\hline$J_{B G}$ & 73 & $(45.34)$ & 199 & $(122.32)$ & 158 & $(97.16)$ & 160 & $(98.70)$ & 156 & $(96.18)$ \\
\hline$\overline{G S_{B G}}$ & & - & 61 & $(41.56)$ & 49 & (33.38) & 44 & $(29.80)$ & 42 & $(28.68)$ \\
\hline$I L U_{B G}$ & 25 & $(23.90)$ & 29 & $(27.74)$ & 19 & $(18.34)$ & 16 & $(15.58)$ & 16 & $(15.60)$ \\
\hline$M I L U_{B G}$ & 18 & $(17.40)$ & 18 & $(17.28)$ & 16 & $(15.56)$ & 15 & $(14.92)$ & 15 & $(14.66)$ \\
\hline \multicolumn{11}{|l|}{$n=1$} \\
\hline$A B F$ & $\overline{3}$ & $(32.70)$ & 39 & $(335.30)$ & 25 & $(352.04)$ & 11 & $(176.64)$ & 9 & $(155.34)$ \\
\hline$J_{B G}$ & 51 & $(31.90)$ & 137 & $(84.84)$ & 163 & $(101.14)$ & 247 & $(152.82)$ & 196 & $(121.28)$ \\
\hline$G S_{B G}$ & $\overline{58}$ & $(39.54)$ & 60 & $(40.86)$ & 60 & $(40.82)$ & 60 & $(40.82)$ & 59 & $(40.14)$ \\
\hline$I L U_{B G}$ & 18 & $(17.54)$ & 22 & $(21.36)$ & 22 & $(21.32)$ & $\overline{21}$ & $(20.42)$ & 19 & $(18.50)$ \\
\hline$M I L U_{B G}$ & 17 & (16.62) & 17 & $(16.64)$ & 17 & $(16.60)$ & 17 & $(16.66)$ & 17 & $(16.62)$ \\
\hline \multicolumn{11}{|l|}{$\eta=10$} \\
\hline$\overline{A B F}$ & 3 & $(26.42)$ & 25 & $(190.78)$ & $\overline{69}$ & $(639.62)$ & $\overline{29}$ & $(382.96)$ & 21 & $(300.48)$ \\
\hline$J_{B G}$ & 50 & $(31.34)$ & & - & 115 & $(71.40)$ & 189 & $(117.44)$ & 200 & $(123.78)$ \\
\hline$\overline{G S}_{B G}$ & 60 & $(40.86)$ & 60 & $(40.82)$ & 60 & $(41.02)$ & 60 & $(41.80)$ & 59 & $(40.34)$ \\
\hline$I L U_{B G}$ & 19 & $(18.50)$ & 18 & $(17.60)$ & 18 & $(17.64)$ & 19 & $(18.98)$ & 19 & $(18.70)$ \\
\hline$M I L U_{B G}$ & $\overline{17}$ & $(16.64)$ & 17 & (16.62) & 17 & $(16.70)$ & 17 & $(16.66)$ & 17 & $(16.68)$ \\
\hline
\end{tabular}

Table 7: Iterations (Time) for $n=15, \eta=50,100$, varying $\epsilon$

\begin{tabular}{|c|c|c|c|c|c|c|c|c|c|c|}
\hline$\eta=\overline{50}$ & \multicolumn{2}{|c|}{$c=0$} & \multicolumn{2}{|c|}{$\epsilon=1$} & \multicolumn{2}{|c|}{$c=10$} & \multicolumn{2}{|c|}{$c=50$} & \multicolumn{2}{|c|}{$c=100$} \\
\hline$A B F$ & 3 & (25.72) & 11 & $(83.66)$ & & $(345.38)$ & 59 & $(553.30)$ & 43 & $(475.40)$ \\
\hline$J_{B G}$ & $\overline{45}$ & $(28.22)$ & 110 & $(68.34)$ & & - & 112 & $(69.50)$ & 157 & $(97.36)$ \\
\hline$G S_{B G}$ & $\overline{59}$ & $(40.20)$ & 59 & $(40.24)$ & 59 & $(40.14)$ & 59 & $(40.28)$ & 59 & $(40.14)$ \\
\hline$I L U_{B G}$ & 20 & $(19.42)$ & 20 & (19.42) & 20 & $(19.52)$ & 19 & $(18.56)$ & 19 & $(18.46)$ \\
\hline$M I L U_{B G}$ & 17 & $(16.60)$ & 17 & (16.58) & 17 & $(16.60)$ & $\overline{17}$ & $(16.76)$ & 17 & $(16.64)$ \\
\hline \multicolumn{11}{|l|}{$\eta=100$} \\
\hline$A \bar{B} F$ & 3 & $(25.32)$ & 7 & $(54.50)$ & 27 & $(194.24)$ & 63 & $(523.82)$ & 55 & $(528.20)$ \\
\hline$J_{B G}$ & & $(27.60)$ & 55 & (34.48) & & 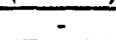 & 198 & $(122.92)$ & 112 & $(71.32)$ \\
\hline$G S_{B G}$ & $\overline{59}$ & $(40.16)$ & 59 & $(40.26)$ & 59 & $(40.20)$ & 59 & $(40.18)$ & 59 & $(40.16)$ \\
\hline$I L U_{B G}$ & $\overline{20}$ & $(19.48)$ & 20 & $(19.54)$ & 20 & (19.34) & 20 & $(19.44)$ & 20 & $(19.46)$ \\
\hline$M I L U_{B G}$ & & $(16.78)$ & 17 & $(16.62)$ & 17 & $(16.68)$ & 17 & $(16.68)$ & 17 & $(16.62)$ \\
\hline
\end{tabular}




\section{Summary}

In this paper various iterative methods and preconditioners were applied to the solution of a model system of coupled elliptic equations. Experimental results were presented for a range of values for the two parameters of the coupled system.

The hybrid method ABF was by far the most robust and, typically, the most time efficient method among the non-accelerated methods studied. Among the preconditioners employed, the block ILU and MILU methods based on "by grid point" ordering were seen to be the most efficient and robust over a wide range of values for the parameters.

This is noteworthy because the incomplete LU preconditioners ordered "by grid point" outperformed most methods even when the coupling between variables on the same grid point was very weak. This behavior, however, follows from the physics involved in coupled systems. Even in a mildly coupled system, the physical effects of the coupling are crucial to the behavior of the system. So, it is not surprising that an iterative method or preconditioner that utilizes the coupling will have an intrinsic advantage over techniques that treat each variables separately. Such behavior was seen in [2] for a on^-dimensional coupled system. Similar behavior is expected in threedimensional problems as well. 


\section{References}

[1] J.M.C. Aarden and K.-E. Karlsson, Preconditioned CG-type Methods for Solving the Coupled System of Fundamental Semiconductor Equations, BIT 29 (1989), pp. 916-937.

[2] R.E. Bank, T.F. Chan, W.M. Coughran, Jr. and R.K. Smith, The AlternateBlock-Factorization Procedure For Systems of Partial Differential Equations, BIT 29 (1989), pp. 938-954.

[3] W. Fichtner, D.J. Rose, and R.E. Bank, Semiconductor Device Simulation, IEEE Trans. Electron Devices, Vol. 30, No. 9, Sept. 1983.

[4] P. Concus, G.H. Golub and G. Meurant, Block preconditioning for the conjugate gradient method, SIAM J. Sci. Stat. Comp. 6 (1985), pp. 220-252.

[5] J. M. Donato, Iterative Methods for Scalar and Coupled Systems of Elliptic Equations, UCLA CAM Report 91-20, Sept. 1991.

[6] G.H. Golub and C.F. Van Loan, Matrix Computations, Johns Hopkins University Press, 1989.

[7] L.A. Hageman and D.M. Young, Applied Iterative Methods, Academic Press, New York, 1981.

[8] D.E. Keyes, and W.D. Gropp, Domain-Decomposable Preconditioners for SecondOrder Upwind Discretizations of Multicomponent Systems, Technical Report, Mathematics and Computer Science, Preprint MCS-P187-1090, Argonne National Lab, Oct. 1990.

[9] J.A. Meijerink and H.A. van der Vorst, An iterative solution method for linear systems of which the coefficient matrix is a symmetric $M$-matrix, Math. Comp. 31 (1977), pp. 148-162.

[10] J.M. Ortega, Numerical Analysis: A Second Course, SIAM, 1990.

[11] Y. Saad and M.H. Schultz, GMRES: A generalized minimal residual algorithm for solving nonsymmetric linear systems, SIAM J. Sci. Stat. Comp. 7, No. 3, pp. 856-869.

[12] R.S. Varga, Matrix Iterative Analysis, Prentice-Hall, Englewood Cliffs, New Jersey, 1971.

[13] D.M. Young, Iterative Solution of Large Linear Systems, Academic Press, Inc., 1971. 


\section{INTERNAL DISTRIBUTION}

\author{
1. B. R. Appleton \\ 2-3. T. S. Darland \\ 4. E. F. D'Azevedo \\ 5-9. J. M. Donato \\ 10. J. J. Dongarra \\ 11. G. A. Geist \\ 12. L. J. Gray \\ 13. M. R. Leuze \\ 14. E. G. Ng \\ 15. C. E. Oliver \\ 16. B. W. Peyton \\ 17-21. S. A. Raby
}

22. C. H. Romine

23. T. H. Row an

24. B. D. Semeraro

25. W.A. Shelton

26-30. R. F. Sincovec

31-35. R. C. Ward

36. P. H. Worley

37. Central Research Library

38. ORNL Patent Office

39. K-25 Appl Tech Library

40. Y-12 Technical Library

41. Lab Records Dept - RC

42-43. Laboratory Records Dept

\section{EXTERNAL DISTRIBUTION}

44. Steven Ashby, Lawrence Livermore National Laboratory, P.O. Box 808, L-316, Livermore, CA 94551

45. Jesse L. Barlow, Department of Computer Science, Pennsylvania State University, University Park, PA 16802

46. Michael Berry, Department of Computer Science, Ayres Hall, University of Tennessee, Knoxville, TN 37996-1301

47. Roger W. Brockett, Pierce Hall 29 Oxford Street Harvard University Cambridge, MA 02138

48. Daniela Calvetti, Stevens Inst of Technology, Department of Mathematics, Hoboken NJ 07030

49. Tony Chan, Department of Mathematics, University of California, Los Angeles, 405 Hilgard Avenue, Los Angeles, CA 90024

50. Alexandre Chorin, Mathematics Department, Lawrence Berkeley Laboratory, Berkeley, CA 94720

51. James Corones, Ames Laboratory, Iowa State University, Ames, IA 50011

52. Rosa Donat, Dept. Matematica Aplicada i Astronomia, Facultat de Matematiques, C/ Dr. Moliner, 50, 46100 Burjassot (Valencia) Spain

53. Donald J. Dudziak, Department of Nuclear Engineering, 110B Burlington Engineering Labs, North Carolina State University, Raleigh, NC 27695-7909

54. Howard C. Elman, Computer Science Departmeni, University of Maryland, College Park, MD 20742

55. Richard E. Ewing, Department of Mathematics, Texas A\&M University, College Station, TX 77843 
56. James Glimm, Department of Mathematics, State University of New York, Stony Brook, NY 11794

57. Gene H. Golub, Department of Computer Science, Stanford University, Stanford, CA 94305

58. Michael T. Heath, National Center for Supercomputing Applications, 4157 Beckman Institute, University of Illinois, 405 North Mathews Avenue, Urbana, IL 61801-2300

59. Fred Howes, Office of Scientific Computing, ER-7, Applied Mathematical Sciences, Office of Energy Research, Department of Energy, Washington, DC 20585

60. Gary Johnson, Office of Scientific Computing, ER-7, Applied Mathematical Sciences, Office of Energy Research, Department of Energy, Washington, DC 20585

61. Malvyn H. Kalos, Cornell Theory Center, Engineering and Theory Center Bldg., Cornell University, Ithaca, NY 14853-3901

62. Hans Kaper, Mathematics and Computer Science Division, Argonne National Laboratory, 9700 South Cass Avenue, Bldg. 221, Argonne, IL 60439

63. Linda Kaufman, Bell Laboratories, 600 Mountain Avenue, Murray Hill, NJ 07974

64. David Keyes, Mechanical Engineering Dept., Yale University, P.O. Box 2159, Yale Station, New Haven, CT 06520-2159

65. David Kincaid, Center for Numerical Analysis, RLM 13-150, Austin, TX 78712

66. Peter D. Lax, Courant Institute of Mathematical Sciences, New York University, 251 Mercer Street, New York, NY 10012

67. James E. Leiss, Rt. 2, Box 142C, Broadway, VA 22815

68. Juan Meza, Sandia National Laboratories, Division 8211, P.O. Box 969, Livermore, CA 94551-0969

69. Neville Moray, Department of Mechanical and Industrial Engineering, University of Illinois, 1206 West Green Street, Urbana, IL 61801

70. David Nelson, Director of Office of Scientific Computing Staff, ER-7, Applied Mathematical Sciences, Office of Energy Research, Department of Energy, Washington, DC 20585

71. Dianne P. O'Leary, Computer Science Department, University of Maryland, College Park, MD 20742

72. Julia Olkin, Remote Measurement Lab., SRI International \#301-66, 333 Ravenswood Ave., Menlo Park, CA 94025-3493

73. Beth Ong, University of California, San Diego, Department of Mathematics, 9500 Gilman Drive, La Jolla, CA 92093-0112

74. James M. Ortega, Department of Applied Mathematics, Thornton Hall, University of Virginia, Charlottesville, VA 22901

75. Jesse Poore, Department of Computer Science, Ayres Hall, University of Tennessee, Knoxville, TN 37996-1301

76. Lothar Reichel, Kent State University, Dept. of Mathematics and Computer Science, Kent, OH 44242 
77. Horst Simon, Mail Stop T045-1, NASA Ames Research Center, Moffett Field, CA 94035

78. G. W. Stewart, Computer Science Department, University of Maryland, College Park, MD 20742

79. Charles Tong, Dept. 8210, Sandia National Labs., Livermore, CA 94551-0969

80. Lloyd Nicholas Trefethen, Cornell University, Dept. of Computer Science, Ithaca, NY 14853

81. Mary F. Wheeler, Rice University, Department of Mathematics, P.O. Box 1892, Houston, TX 77251

82. David Young, University of Texas, Center for Numerical Analysis, RLM 13.150, Austin, TX 78731

83. Office of Assistant Manager for Energy Research and Development, Department of Energy, Oak Ridge Operations Office, P.O. Box 2001 Oak Ridge, TN 37831-8600

84-85. Office of Scientific \& Technical Information, P.O. Box 62, Oak Ridge, TN 37831 
$r$

- 

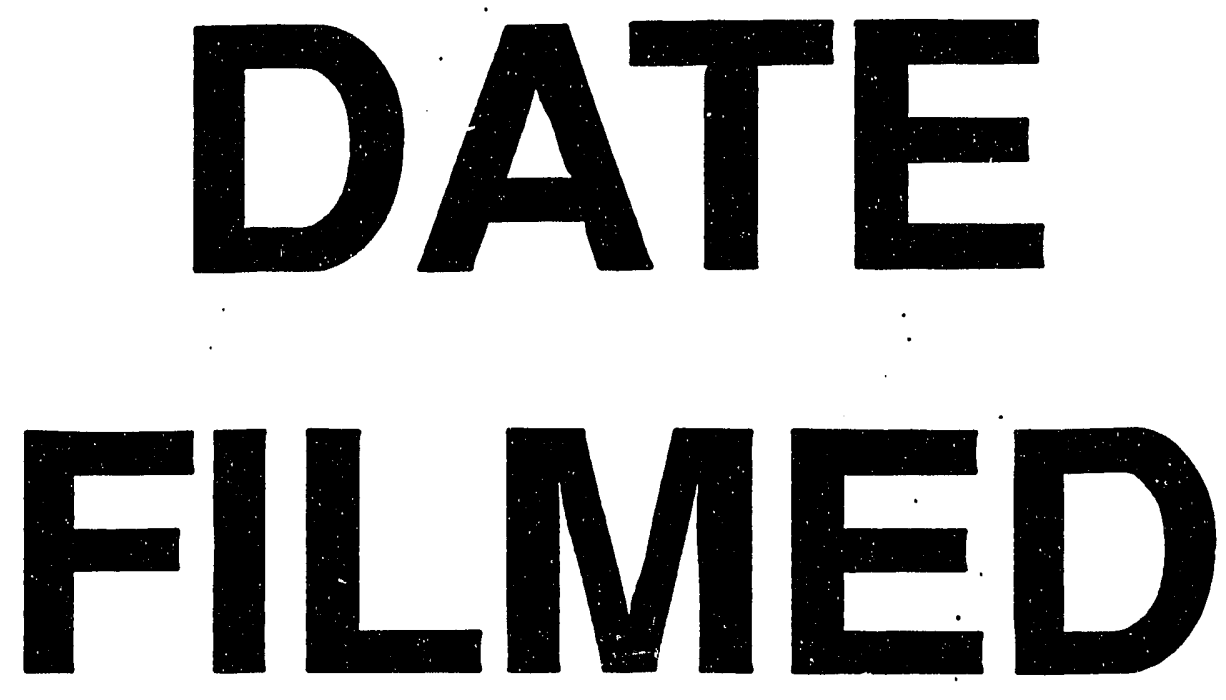

$10 / 15 / 93$
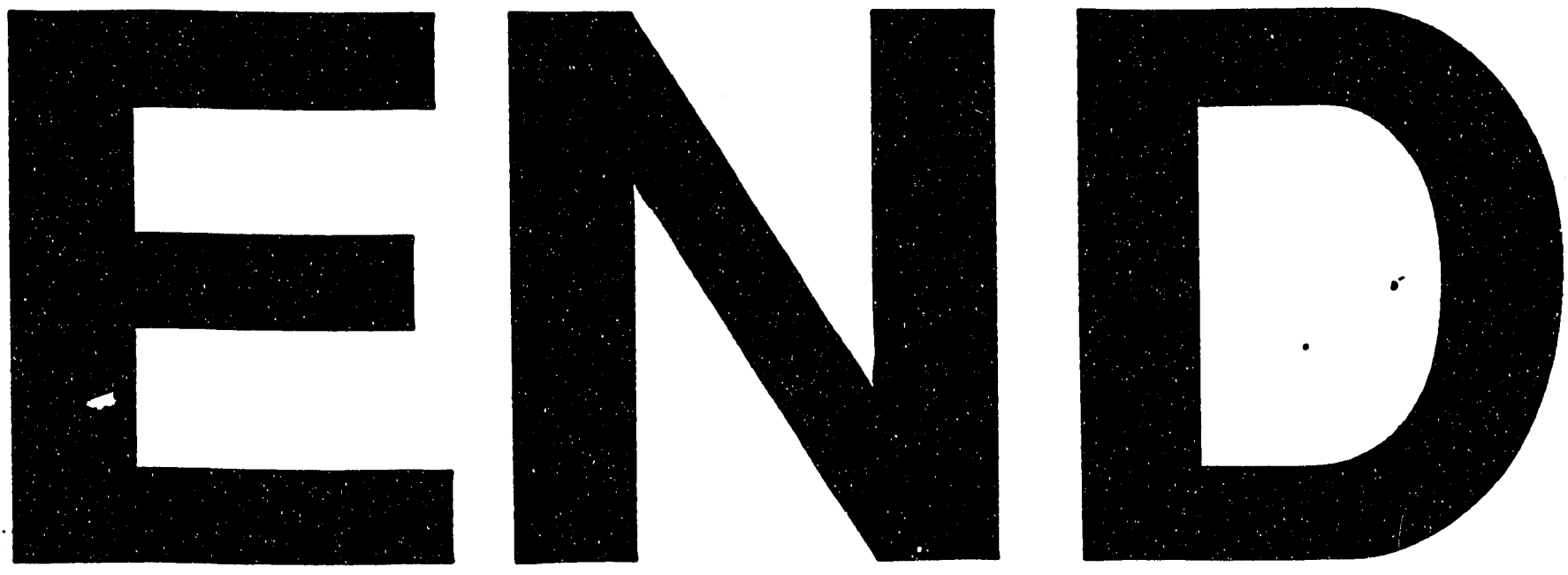
